\title{
About the Energy Interval beyond the Ankle Where the Cosmic Radiation Consists Only of Ultraheavy Nuclei from Zinc to the Actinides
}

\author{
Antonio Codino \\ Dipartimento di Fisica, Università di Perugia and INFN, Perugia, Italy \\ Email: Antonio.codino@pg.infn.it
}

How to cite this paper: Codino, A. (2017) About the Energy Interval beyond the Ankle Where the Cosmic Radiation Consists Only of Ultraheavy Nuclei from Zinc to the Actinides. Journal of Applied Mathematics and Physics, 5, 225-237.

http://dx.doi.org/10.4236/jamp.2017.51020

Received: October 16, 2016 Accepted: January 24, 2017 Published: January 31, 2017

\begin{abstract}
According to recent measurements the tendency of the chemical composition above the ankle is characterized by increasing fractions of intermediate and heavy nuclei and a dominance of light nuclei around the ankle. Calculation of the chemical composition in the range $3.5 \times 10^{18}-5 \times 10^{19} \mathrm{eV}$ according to new principles explains both the rising tendency of the heavy component. The calculation is prolonged to the adjacent interval $5 \times 10^{19}-2.4 \times 10^{21} \mathrm{eV}$ using the same theoretical background and some features of the observed cosmic-ray spectrum. It results that above the energy of $6.7 \times 10^{20} \mathrm{eV}$, where the flux is estimated to be $1.8 \times 10^{-30}$ particles $/ \mathrm{m}^{2} \mathrm{~s} \mathrm{sr} \mathrm{GeV}$, the cosmic radiation consists only of nuclei heavier than Zinc. Measurements of the spectrum of present and past experiments are compared with the calculations.
\end{abstract}

\section{Keywords}

Ultrahigh Energy Cosmic Ray, Chemical Composition, GZK Effect

\section{Introduction}

In the year 2005-2007 the HiRes Collaboration reported unequivocal evidence for a break of the cosmic-ray spectrum close and above to $5 \times 10^{19} \mathrm{eV}$ [1]. This suppression was confirmed by the Auger Collaboration at a significant lower energy [2] in the range $(2-3) 10^{19} \mathrm{eV}$. Presently this fundamental feature of the spectrum can be further investigated by the Telescope Array (hereafter $T A$ ) and Auger Collaborations which operate the two largest instruments gathering data above $10^{19} \mathrm{eV}$.

The TA experiment located in Utah, North America, has a collecting area of about $700 \mathrm{Km}^{2}$ and is deployed in the historical site of the Fly's Eye experiment which first recorded the florescence light of air showers in order to measure the 
energy of primary cosmic nuclei. The technique improves the energy resolution achievable otherwise.

The Auger experiment located in Argentina, South America, has a collecting area of about $3000 \mathrm{Km}^{2}$. The difference in the collecting areas of the two instruments is reflected in the error bars of the measurements. In fact both instruments use the same hybrid technique which jointly exploits the florescence light yield and the muon density at ground of giant air cascades. Although the data samples collected by the Auger apparatus for measuring the energy spectrum and the chemical composition have an intrinsic superior statistical precision, this study necessitates the outcomes of both experiments.

The calculation of the cosmic-ray spectrum in the range $10^{19}-2.4 \times 10^{21} \mathrm{eV}$ presented in this paper is based on two empirical and one theoretical inputs designated by A, B and C. The limited size of this paper impedes a critical examination of the empirical inputs $\mathrm{A}$ and $\mathrm{B}$. Consequently they are concisely summarized by two statements: (A) the chemical composition of the cosmic radiation evolves from light to heavy in the range $5 \times 10^{18}-10^{20} \mathrm{eV}$ [3] [4] [5] [6]; (B) there is a suppression in the energy spectrum above $2.6 \times 10^{19} \mathrm{eV}$ [1] [2] with respect to a power-law extrapolation from the vast, adjacent, lower energy band, for instance the energy decade $2.6 \times 10^{18}-2.6 \times 10^{19} \mathrm{eV}$ [7].

Let us anticipate that the energy scales of the instruments are of critical importance for the validation of the energy spectrum computed in this paper above $2.6 \times 10^{19} \mathrm{eV}$ because imperfections in the calibration of the energy scales affect also the observed fluxes. The Auger apparatus in the hybrid mode of operation at $10^{19} \mathrm{eV}$ has an energy resolution of about 15 per cent and 10 per cent at $10^{20}$ $\mathrm{eV}$. The TA instrument has a comparable energy resolution. As a matter of fact, if the energies reported in the published measurements are regarded as real and not fleeting, there was (2007) and there is (2016) an evident mismatch in the energy scales of the HiRes, Auger and TA instruments. Probably the inter-experiment calibration is not resolvable by a rigid shift in the range $10^{19}-10^{20} \mathrm{eV}$ but involves non linear adjustments of the energy scales.

\section{The Power Law of the Energy Spectrum of the Cosmic Radiation with a Single Index}

A brief description of the third input $C$ follows. Two decades of research via numerical simulation of the properties of Galactic cosmic rays have led to the explanation of the knee and ankle of the energy spectrum of the cosmic radiation [8]. The knee and the ankle are effects caused by the particle transport in the Galaxy (propagation effects) and not by the acceleration mechanism. This explanation is achievable only by introducing a notable assumption called Principle of Constant Indices [9] which states:

The physical process accelerating cosmic rays in the Galaxy releases particles with an energy spectrum featured by a power law and a constant index of $2.67 \pm$ 0.05 in the energy range $10^{9}-5 \times 10^{19} \mathrm{eV}$.

Cosmic-ray propagation in the Galaxy has a negligible effect (see Chapter 17 
of ref. 10) on the index inherent the acceleration process. Why this assumption is called principle is justified elsewhere [9].

The cosmic-ray spectrum shown in Figure 1 in the limited range $10^{9}-2.6 \times$ $10^{19} \mathrm{eV}$ is comprised between two rails, the blue lines, which differ by a factor 38 marked in Figure 1. The theoretical framework which explains the knee and ankle features also provides the characteristic gap expressed by the factor 38 [8].

Measurements of the energy spectra of 12 individual cosmic nuclei in the preknee region $10^{11}-10^{15} \mathrm{eV}$ indicate that the spectral indices are compatible with a common value of $2.67 \times 0.05$ [11]. Above the ankle energy the all-particle spectrum measured by Haverah Park, Yakutsk, Fly's Eye, HiRes, AGASA, Auger and TA experiments is also compatible with the common index of $2.67 \times 0.05$ observed in the preknee energy region (see for example Figure 2 of ref. [10]).

Presently (2016) the physical process in the Galaxy accelerating cosmic rays in the range $10^{9}-2.6 \times 10^{19} \mathrm{eV}$ is unknown, nevertheless it has some identified fea-

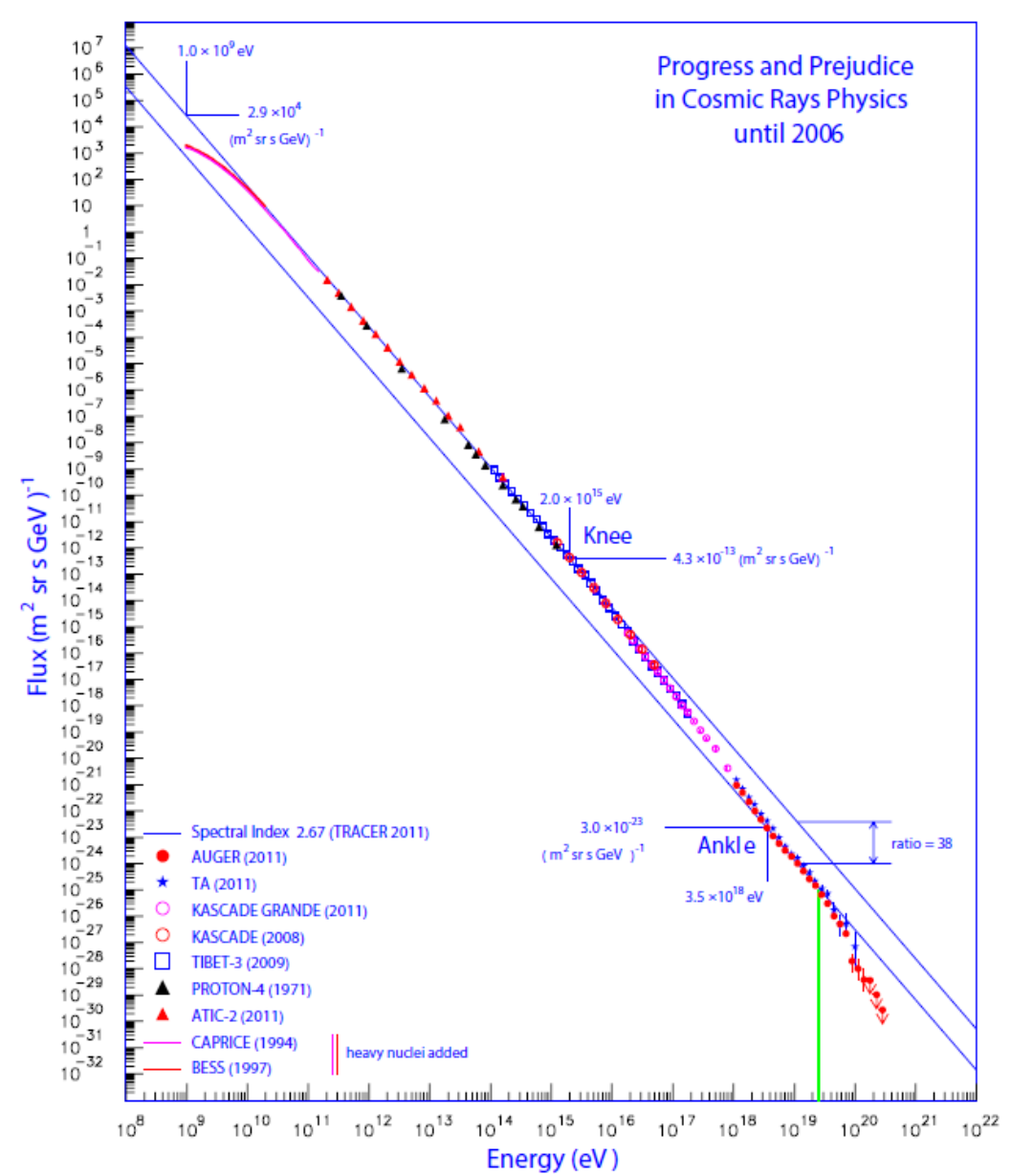

Figure 1. Energy spectrum of the cosmic radiation between $10^{8}$ and $2 \times 10^{20} \mathrm{eV}$ measured by many experiments in more than 60 years. Up to the energy $2.6 \times 10^{19} \mathrm{eV}$ the spectrum lies between two rails featured by an index of 2.67 and separated by a factor 38 . The four major marks of the spectrum are indicated : the arc of the solar modulation below $10^{10}$ $\mathrm{eV}$, the knee above $3.0 \times 10^{15} \mathrm{eV}$, the ankle at $3.5 \times 10^{18} \mathrm{eV}$ and the break at $2.6 \times 10^{19} \mathrm{eV}$ indicated by a vertical green segment. References to the data of the figure are elsewhere [10]. 
tures [9] [10]: (1) it is distributed in space; (2) the accelerated particles obey a power law compatible with a single index of $2.67 \pm 0.05$ [11]; (3) it operates in the range $10^{9}-2.0 \times 10^{20} \mathrm{eV}$. For conciseness in this paper the ensemble of these features is designated by Galactic Accelerator. Thus the Principle of Constant Indices [9] has been rephrased here by the properties of the Galactic Accelerator.

Other parameters of the Galactic Accelerator, conceivable a priori, could be the maximum energy of operation $\mathrm{E}_{\max }$ and the efficiency of the acceleration cycle versus energy denoted here $F$. The efficiency may be a function of some variables such as the energy, the time interval elapsed between the birth and the extinction of the cosmic ray, the nuclei abundances at the injection stage of the acceleration cycle, and others. The acceleration cycle denotes a sequence of subprocesses that convert nuclei of the quiescent matter at the injection stage, up to the highest observed energies of $3 \times 10^{20} \mathrm{eV}$ [1] [2] and eventually beyond this empirical limit.

The Galactic Accelerator is expected to attain $\mathrm{E}_{\max }$ above $2.6 \times 10^{19} \mathrm{eV}$ since below this energy the spectrum conforms perfectly to a power law with a parameter of 2.67 (see Figure 36 ref. 10). The tentative energy $\mathrm{E}_{\max }$ reached by Uranium nuclei is likely $2.4 \times 10^{21} \mathrm{eV}$ which is regarded in this paper as the extreme limit to the Galactic Accelerator.

\section{The Failure of Particle Injection to the Galactic Accelerator}

Data useful for the following inference are the flux and the chemical composition in the range $10^{19}-10^{20} \mathrm{eV}$ condensed in the statements $(\mathrm{A})$ and $(\mathrm{B})$ in the Introduction. A scheme of the logical paths of the inference is summarized in

Figure 2. Simplicity is an ingredient of the reasoning and intervenes in more

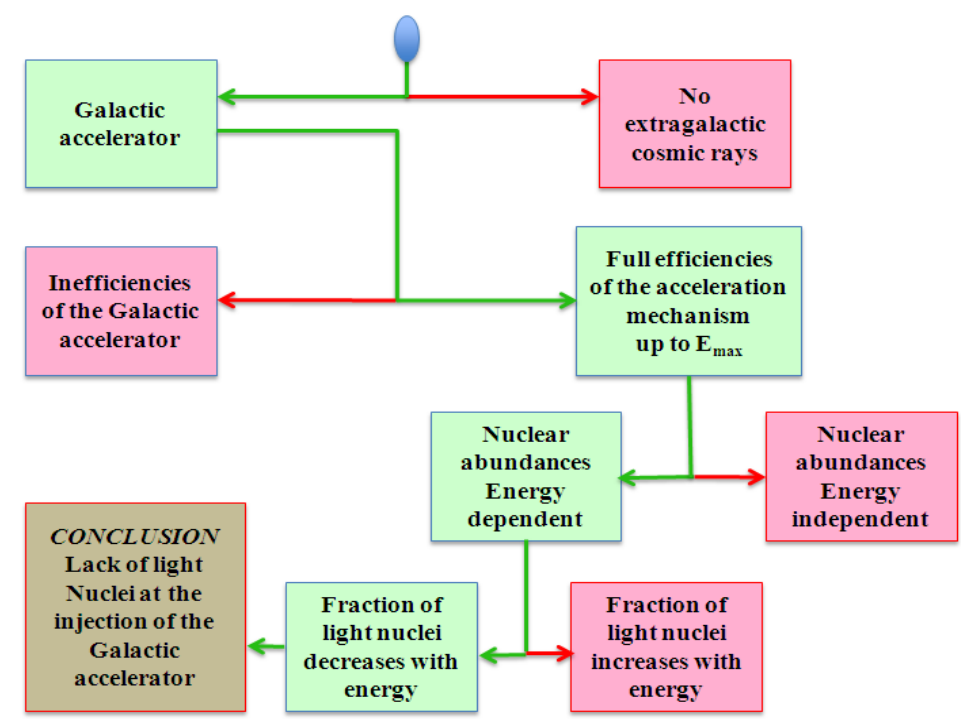

Figure 2. Logical scheme with four biforcation levels based on experimental data (energy spectrum and chemical composition) leading to the conclusion that quiescent nuclei are suppressed at the injection of the galactic accelerator (see also Figure 3) above the energy threshold $\mathrm{E}_{\mathrm{LI}}(\mathrm{Z})$ proportional to the atomic number $\mathrm{Z}$ of the nucleus. 
than one link of the logical chain. The intrinsic acceleration process is also assumed not to alter the chemical composition at the injection, which is a common assumption recurrent in the literature.

A priori any incipient deviation from a power-law spectrum with a single parameter could be a depression or an enhancement. The data clearly manifest a depression above the energy $2.6 \times 10^{19} \mathrm{eV}$ marked by a vertical green segment in Figure 1 . This particular energy is designated by $\mathrm{E}_{\mathrm{LI}}(\mathrm{H})$ and shortly by $\mathrm{E}_{\mathrm{LI}}$ where $\mathrm{H}$ is for Hydrogen or protons and LI for Lack of particle Injection to the Galactic Accelerator. These wordings are justified below.

According to the Auger Collaboration the flux depression is halved with respect to a power-law extrapolation with a single parameter at the

energy of $(4.01 \pm 0.21) \times 10^{19} \mathrm{eV}$ [13] which is quite consistent with $\mathrm{E}_{\mathrm{LI}}=2.6 \times$ $10^{19} \mathrm{eV}$ being this value the lowest energy where the deviation manifests itself (see Figure 36 ref. 10).

Since the hypothetical extragalactic component would yield an enhancement relative to the extrapolation, the observed spectrum rules out the extragalactic component (level 1 of Figure 2). It follows that the Galactic Accelerator is still operating at this energy but a subprocess of the entire acceleration cycle initiates to fail, or equivalently, that the Galactic Accelerator is loosing its global efficiency exhibited and demonstrated below $2.6 \times 10^{19} \mathrm{eV}$ via the constant index of 2.67 \pm 0.05 . Since the break in the spectrum at the energy $E_{\mathrm{LI}}$ does exist [1] [2] either the intrinsic acceleration process is becoming inefficient or nuclear abundances are changing with the energy. The constraint of the simplicity dictates that the chemical composition and the acceleration efficiency do not vary simultaneously in the same energy range.

Notice the following important circumstance about the chemical composition of the cosmic radiation around and above to $10^{19} \mathrm{eV}$. The fractions of cosmic nuclei above $10^{19} \mathrm{eV}$ cannot change by nuclear interactions as they do at lower energies. In fact the matter density in the interstellar medium is about $1 \mathrm{~g} / \mathrm{cm}^{3}$. A nucleus crossing the Milky Way Galaxy at very high energy accumulates an average grammage between 6 to 8 millig/ $\mathrm{cm}^{2}$. Since interaction cross sections are known, it follows that the nuclear abundances cannot change by significant amounts. Due to this circumstance the fractions of nuclei at $10^{19} \mathrm{eV}$ cannot be very different from those measured in the preknee energy region. Detailed calculations of the chemical composition in the range $10^{12}-10^{19} \mathrm{eV}$ confirm this crude estimate (see Figure 7 of The new horizon disclosed by the measurement of the chemical composition of the cosmic radiation above the ankle energy, Proceedings of Science, ICRC 2015, paper 466, by A. Codino).

The measurements of the Auger and TA experiments indicate that the nuclei fractions change with the energy in the range $10^{19}-10^{20} \mathrm{eV}$ (level 3 in Figure 2). Moreover they change in a very selective manner: the fraction of heavy ions augments with energy, or equivalently, the fraction of light ions decreases with energy (level 4 in Figure 2). The trend of the chemical composition with energy is certain but the absolute fractions of individual nuclei composing the cosmic 
radiation are still not known. The simple conclusion to be drawn is that: (1) the acceleration mechanism performs with the same efficiency above $\mathrm{E}_{\mathrm{LI}}=2.6 \times 10^{19}$ $\mathrm{eV}$; and (2) the relative abundances of cosmic nuclei at the sources, before acceleration, change with the energy.

Major alternatives excluded by data as structured in the scheme of Figure 2 in the energy interval $\mathrm{E}_{\mathrm{LI}}-\mathrm{E}_{\max }$ are: (first alternative) (1) the efficiency of the acceleration process changes with the energy and (2) the relative abundances of the cosmic nuclei are energy independent; (second alternative) (1) the efficiency of the acceleration process does not change with the energy and (2) the relative abundances of cosmic nuclei are energy independent.

According to the conclusion given in Figure 2 the cosmic-ray flux is expected to abruptly fall as the energy increases and then to stabilize in plateaux (staircase pattern) as shown in Figure 3. The gap between adjacent plateaux are directly proportional to the abundances of the nuclear species in the interval, $1 \leq \mathrm{Z} \leq 92$. Thus the observed flux above $\mathrm{E}_{\mathrm{LI}}$ can deviate from the lower rail in Figure 1 by discrete amounts; amounts related to the fractions of nuclei composing the cosmic radiation. After the flux fall of a given nucleus, the spectrum regains the same slope of 2.67. Virtually each nucleus gives an intensity step. As the energy increases the lighter nuclear species disappear and the total flux grows thinner and thinner (see Figure 3).

\section{The Predicted Energy Spectrum in the Range $10^{19}-2.4 \times 10^{21} \mathrm{eV}$}

The determination of the energy spectrum above $\mathrm{E}_{\mathrm{LI}} \equiv \mathrm{E}_{\mathrm{LI}}(\mathrm{H})=2.6 \times 10^{19} \mathrm{eV}$ requires two additional parameters: (I) the abundances of cosmic-ray nuclei around $10^{19} \mathrm{eV}$ at the injection; (II) the rule by which cosmic-ray nuclei are filtered at the injection.

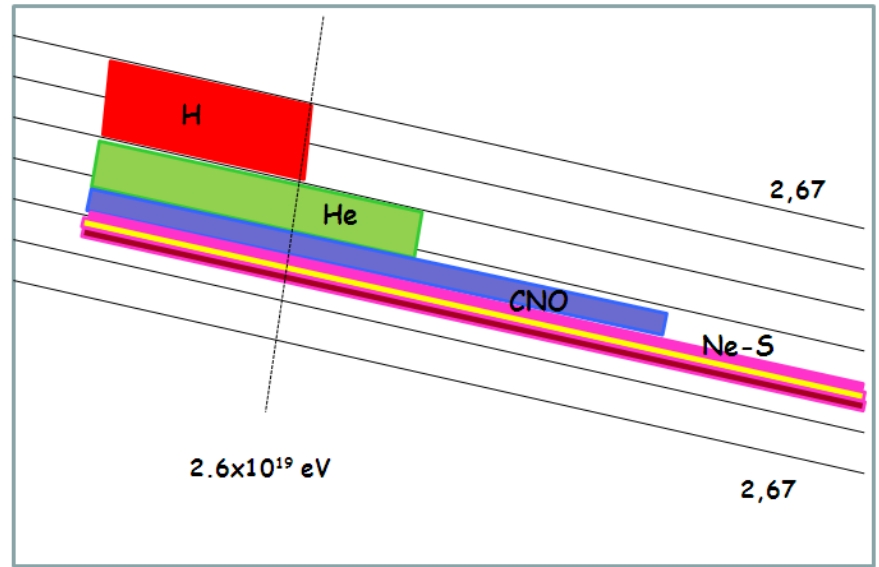

Figure 3. Illustration of the mechanism giving rise to a staircase pattern in the energy spectrum. The mechanism is designated in this paper by lack of particle injection to the Galactic Accelerator and the lower energy where it manifests itself is $\mathrm{E}_{\mathrm{LI}}=2.6 \times 10^{19} \mathrm{eV}$. As far as the energy increases above $\mathrm{E}_{\mathrm{LI}}$ quiescent atoms at the injection are somehow filtered, initiating with the lightest nuclei, i.e. protons and terminating with the heaviest one, Uranium at the energy $\mathrm{E}_{\mathrm{LI}}(\mathrm{U})=92 \times \mathrm{E}_{\mathrm{LI}}(\mathrm{H})=2.4 \times 10^{21} \mathrm{eV}$. 
The fractions of nuclei of the cosmic radiation normalized to Iron adopted in this calculation are: $\mathrm{H} 0.40$, He 0.22, CNO 0.17, Ne-S 0.10, F-Ca, 0.012, Sc-Ni .089 and Zc-Ge $7.4 \times 10^{-4}$ based on a variety of measurements [14] [15] [16]. They are expressed in total energy per particle and not in energy per nucleon. This unit is appropriate for very high energies. Nuclei are bunched in small groups with adjacent atomic numbers to simplify the calculations.

The relative amounts of nuclei at the injection do not suffice to calculate the energy spectrum because the particular energy of a nucleus $Z$ for which the injection process is hampered, is not assigned. The suppression mechanism delineated in Figure 3 only implies the disappearance of lighter nuclei before the heavier ones, as the energy increases. The simplest rule reflecting the fact that the chemical composition is becoming heavier above $10^{19} \mathrm{eV}$ is to admit that nuclei of atomic number $Z$ are depleted above the threshold energies $E_{\mathrm{II}}(Z)$, that is, $E_{\mathrm{LI}}(\mathrm{Z})=\mathrm{Z} \times \mathrm{E}_{\mathrm{LI}}(\mathrm{H})$. The implication is that above the energy $\mathrm{E}_{\mathrm{LI}}(\mathrm{Z})$ the element $Z$ is not available as cosmic-ray source matter for any reason whatsoever. According to this linear relationship between $\mathrm{E}$ and $\mathrm{Z}$, Helium is expected to be depleted above the energy, $\mathrm{Z} \times \mathrm{E}_{\mathrm{LI}}(\mathrm{H})=5.2 \times 10^{19} \mathrm{eV}$, Nitrogen above $1.75 \times 10^{20}$ $\mathrm{eV}$, Silicon above $3.5 \times 10^{20} \mathrm{eV}$, Fe nuclei above $6.7 \times 10^{20} \mathrm{eV} \mathrm{eV}$ and so on. If the rule were a linear relationship between $\mathrm{E}$ and $\mathrm{A}$, e.g. $\mathrm{E}_{\mathrm{II}}(\mathrm{Z})=\mathrm{A} \times \mathrm{E}_{\mathrm{II}}(\mathrm{H})$ the incipient energies for the flux fall would change accordingly ( $\mathrm{A}$ is the atomic weight of the nucleus).

The predicted spectrum in the range $10^{19}-2.3 \times 10^{20} \mathrm{eV}$ is given in Figure 4 (green squares) along with the positions of the threshold energies $E_{\mathrm{LI}}(\mathrm{Z})$ of the

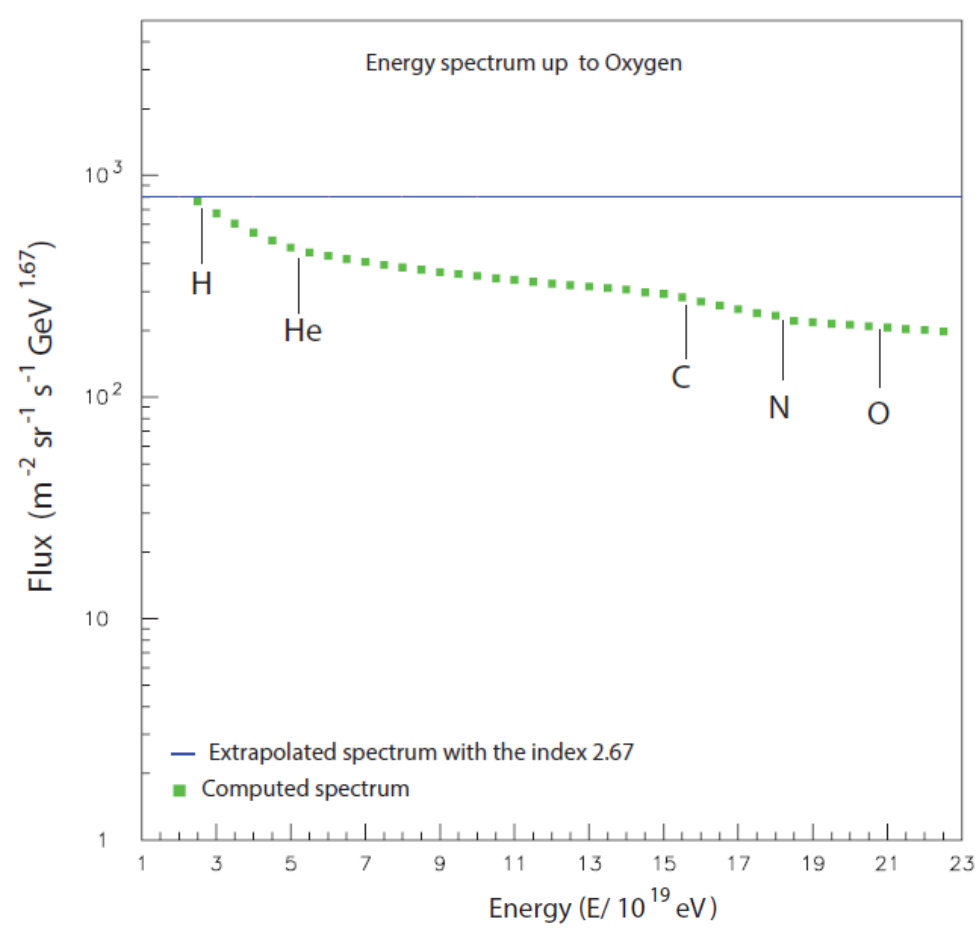

Figure 4. Computed spectrum up to the energy of $2.3 \times 10^{20} \mathrm{eV}$ where the Oxygen depletion is expected to take place. The horizontal blue line is the extrapolated spectrum based on the Auger data [7] in the energy interval $10^{18}-10^{19} \mathrm{eV}$. 
abundant elements ( $\mathrm{H}, \mathrm{He}, \mathrm{C}, \mathrm{N}$ and $\mathrm{O})$. The observed spectrum is multiplied by $\mathrm{E}^{2.67}$ and the blue line represents the resulting flux and its extrapolation to high energies. The blue line is normalized to the Auger data at the intensity of 798 $\mathrm{par} / \mathrm{m}^{2} \mathrm{~s} \mathrm{srGeV}{ }^{1.67}$ [7] which corresponds to $1.159 \times 10^{-25} \mathrm{par} / \mathrm{m}^{2} \mathrm{~s} \mathrm{sr} \mathrm{GeV}$ in the flux unit reported in Figure 1. Note that this normalization to the Auger data, and not to those of the TA experiment, is arbitrary.

Of course the flux in Figure 4 continues to decrease with the power law, which is the characteristic of the Galactic Accelerator, but there is an additional decrement caused by particle injection failure generating a first break above 2.6 $\times 10^{19} \mathrm{eV}$ (proton depletion).

In Figure 5 the computed spectrum is extended up to $2.4 \times 10^{21} \mathrm{eV}$ where Uranium injection failure is predicted to occur. Above the Fe threshold, $\mathrm{E}_{\mathrm{LI}}(26)$ $=26 \times \mathrm{E}_{\mathrm{LI}}(\mathrm{H})=6.7 \times 10^{20} \mathrm{eV}$ Iron disappears, giving rise to an almost vertical flux fall, due to the paucity of trans-iron nuclei. The trans-Iron break is not out of reach of the planned JIM-EUSO experiment [17].

\section{Comparison between Computed and Observed Spectra}

The spectra measured by TA and Auger Collaborations are compared with the predicted spectrum (green squares) in Figure 6. In order to better focus on the minute aspects of the spectrum, a linear scale of energy is used. Below $8 \times 10^{19}$ $\mathrm{eV}$ the computed spectrum lies between the TA and Auger data and above this

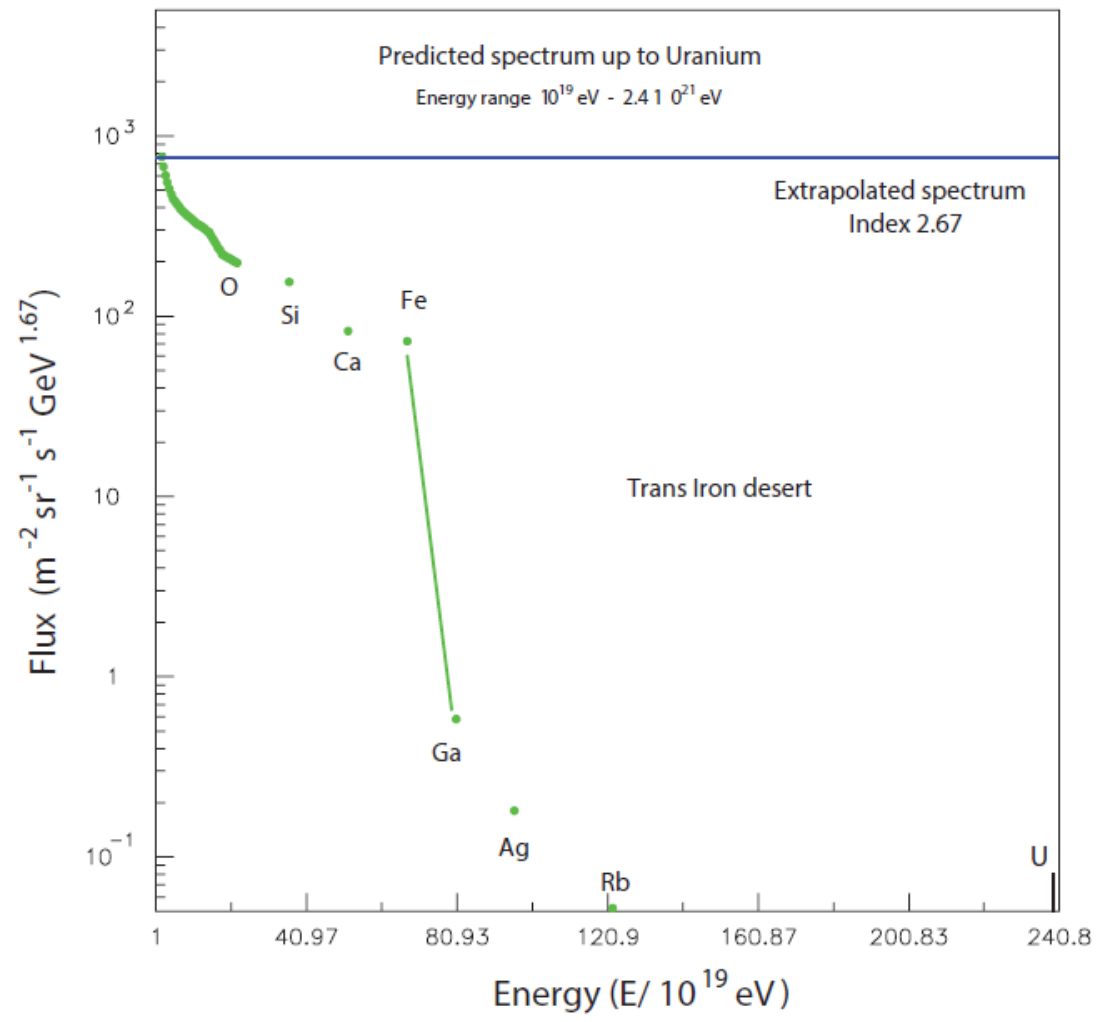

Figure 5. Computed spectrum (green squares) up to the energy of $2.4 \times 10^{21} \mathrm{eV}$ where Uranium depletion is expected to commence. Fluxes above Rubidium nucleus are out of scale, due to their extreme paucity. 


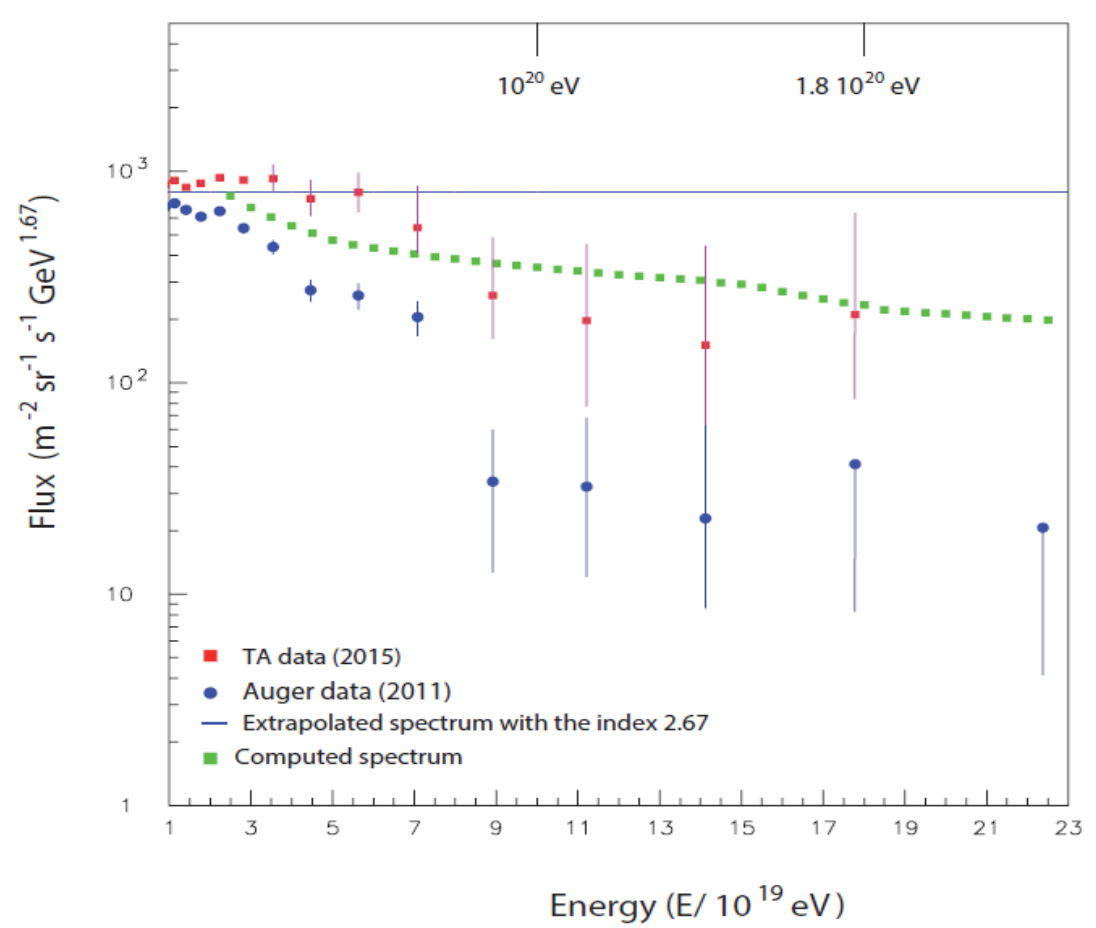

Figure 6. The predicted spectrum (green squares) is compared with the recent measurements of Auger (blue dots) and TA experiments (red squares) [19]. Notice that the last blue dots are upper limits. The horizontal blue line is the extrapolated spectrum based on the Auger data [7] in the energy interval $10^{18}-10^{19} \mathrm{eV}$.

energy is compatible with the TA data while those by Auger are deficient. Notice that the average gap between TA and Auger fluxes above $8 \times 10^{19} \mathrm{eV}$ attains almost an order of magnitude signaling severe problems in the measurement procedures.

Figure 7 and Figure 8 show the comparison with the spectra measured by previous experiments having the largest exposures. An example of the spectrum measured by the Fly's Eye Collaboration, reported in the year 2000 [20] is shown in Figure 7 (black dots). In the half energy decade, between $5 \times 10^{19} \mathrm{eV}$ and $10^{20}$ $\mathrm{eV}$ there is a hint for the proton depletion. Above $10^{20} \mathrm{eV}$ some events are observed and the related flux is slightly above the prediction (green squares).

The spectrum measured by the AGASA Collaboration [21] is shown in Figure 8. With some imagination a small valley is visible in the interval $5 \times 10^{19} \mathrm{eV} 10^{20}$ $\mathrm{eV}$, compared to the extrapolated AGASA spectrum rooted in the range $(1-5) \times$ $10^{19} \mathrm{eV}$ and represented by the black horizontal segment. Data points above $10^{20}$ $\mathrm{eV}$ in Figure 8 exceed the computed spectrum (green squares).

The four quoted experiments together do not disprove the calculations described in this paper. If the comparison is limited to the data collected by the hybrid techniques of the TA and Auger instruments, the predicted flux is too high against the Auger data but not against TA data (Figure 6). Should the comparison include the fluxes reported by AGASA and Fly's Eye experiments, the data are evenly scattered, above and below, around the predicted spectrum and no firm conclusion emerges, neither for rejection nor for validation. The re- 


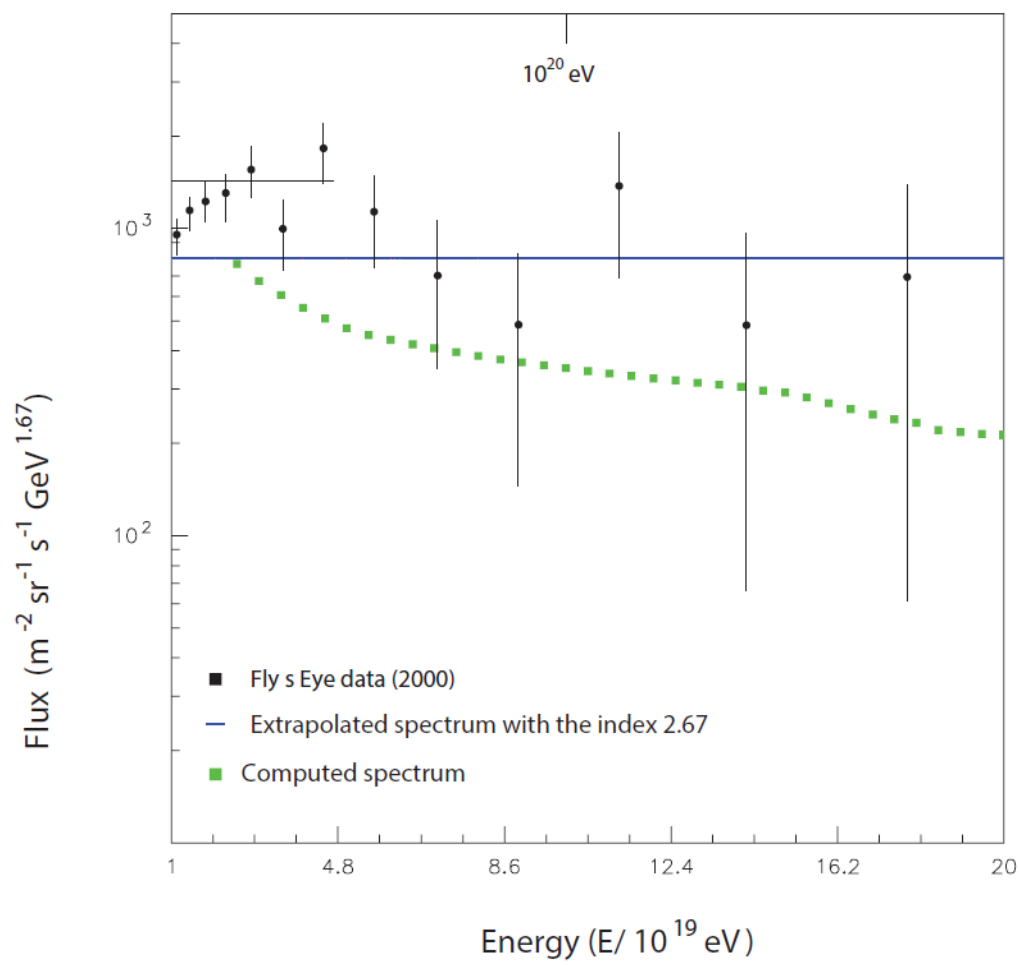

Figure 7. The energy spectrum of Fly's Eye experiment (black dots) [20] is compared with calculation (green squares) in the range $10^{19}-2 \times 10^{20} \mathrm{eV}$.

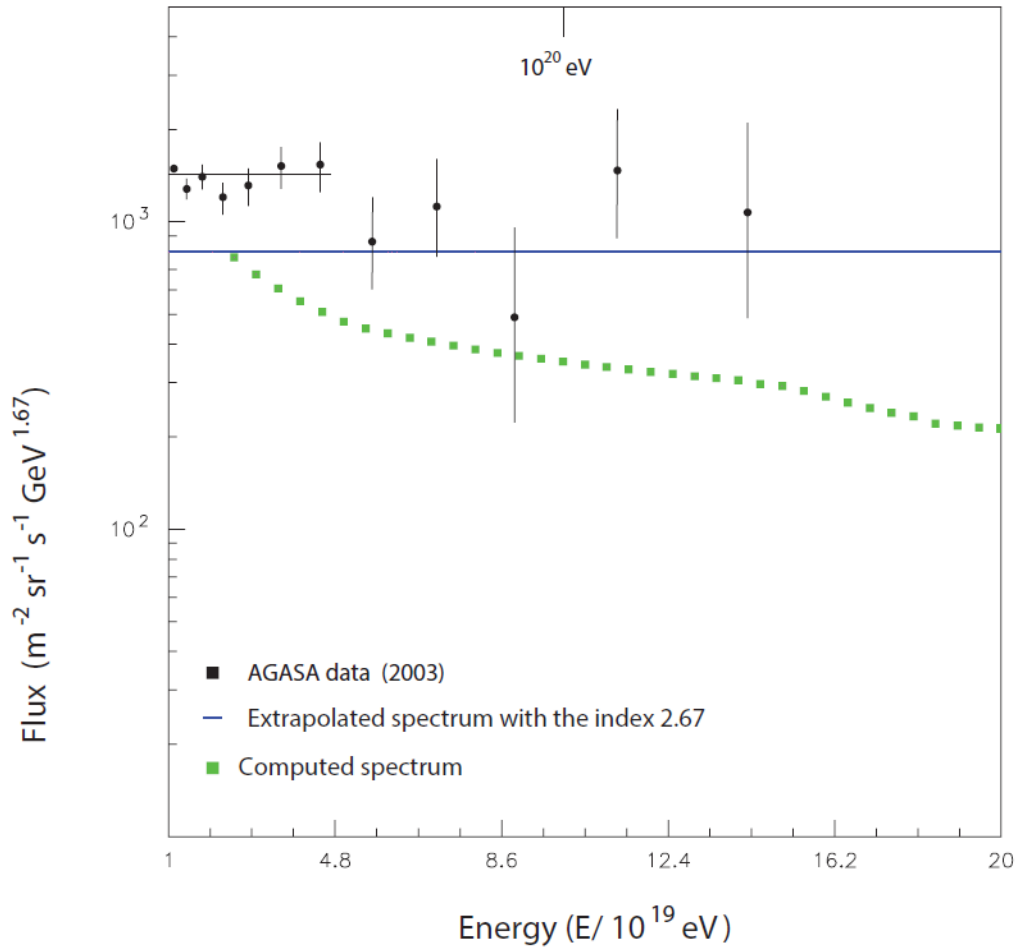

Figure 8. The energy spectrum measured by the AGASA experiment (black dots) [21] is compared with the calculations (green squares). The blue line is normalized to the Auger data [7] while the black horizontal segment is an average value of the AGASA flux in the interval $(1-5) \times 10^{19} \mathrm{eV}$. The highest AGASA data point around $2.3 \times 10^{20} \mathrm{eV}$ is out of scale and does not appear in the figure. 
jection of the predicted spectrum only on the basis of the Auger flux above $8 \times$ $10^{19} \mathrm{eV}$ is premature until the large gap between TA and Auger spectra will not be clarified (Figure 6).

A second fact in favor of the computed spectrum reported in Figure 4 is that the Auger spectrum above $5 \times 10^{19} \mathrm{eV}$ deviates from the extrapolation (blue line Figure 4) not by a power law with a single parameter but by steps, as the Auger data in Figure 9 demonstrate.

An alternative explanation of the observed cosmic-ray spectrum above $10^{19} \mathrm{eV}$ recurrent in the literature is that a hypothetical extragalactic component of the cosmic radiation would suffer a depletion by the impact with the ubiquitous photons of $6.7 \times 10^{-4} \mathrm{eV}$ and density of 411 particles $/ \mathrm{cm}^{3}$. The depletion would commence above $6.0 \times 10^{19} \mathrm{eV}$ according to the original calculations made sixty years ago and is usually described by a power law with a single parameter, much softer than 2.67. This hypothetical phenomenon is known as GZK effect. Let us mention that HiRes, Auger and TA Collaborations have explicitly claimed evidence for the GZK effect [1] [2] [22].

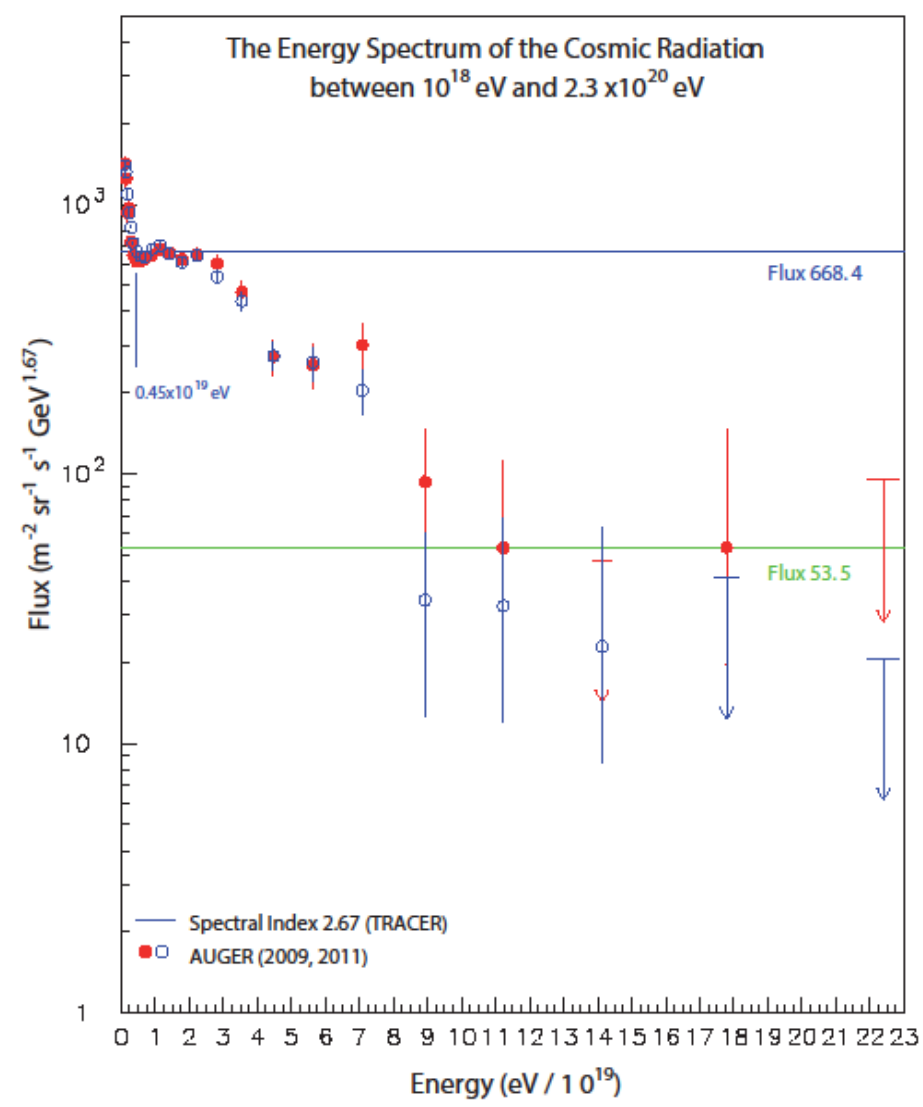

Figure 9. Energy spectrum of the cosmic radiation in a linear scale of energy up to $2.3 \times$ $10^{20} \mathrm{eV}$ measured by the Auger Collaboration [13] [18]. The horizontal blue line represents the extrapolation of the spectrum with a universal index $\gamma_{u}=2.67$. The normalization is slightly different from that of the previous figures. The green horizontal line is a visual guide which marks an arbitrary suppression factor of 12.2 relative to the upper blue line. The data suggest the staircase pattern of the spectrum compatible with the calculation reported in this paper. 
The heavy chemical composition of the cosmic rays above $10^{19} \mathrm{eV}$ makes this alternative explanation quite unlikely as explained elsewhere (The absence of the GZK depression in the energy spectrum of the cosmic radiation, by A. Codino, ICRC 2013, Rio de Janeiro, Brasil).

\section{References}

[1] Abbasi, R.U., et al. (2008) First Observation of the Greisen-Zatsepin-Kuzmin Suppression.

[2] Yamamoto, T., et al. (2007) UHECR Measured at Pierre Auger Observatory and Its Astrophysical Implications.

[3] Unger, M., et al. (2007) Cosmic Ray Composition above $0.4 \mathrm{EeV}$ Using Longitudinal Profiles of Showers Observed at the Pierre Auger Observatory.

[4] Unger, M., et al. (2009) Composition Study with the Pierre Auger Observatory, CRIS 2008 Nuclear Physics B (Proc. Suppl.) 190.

[5] Garcia-Pinto, D. (2011) Measurements of the Longitudinal Development in Air Shower with the Pierre Auger Observatory, Paper 690, Proc. 32nd, Beijing.

[6] Fukushima, M. (2015) Recent Results from Telescope Array.

[7] Aab, A. et al. (2015) Measurement of the Cosmic Ray Spectrum above 4x1018 eV Using Inclined Showers Detected with the Pierre Auger Observatory.

[8] Codino, A. (2017) The Principle of Constant Indices and the Knee and Ankle Features of the Energy Spectrum of the Cosmic Radiation, Submitted for Publication and Available Soon in the Astrophysical World Network.

[9] Codino, A. (2015) The Knee and Ankle Features Derived from the Principle of Constant Indices and the Galactic Accelerator. 34th ICRC, Paper 465, Proceedings of Science, Holland.

[10] Codino, A. (2015) Progress and Prejudice in Cosmic Ray Physics until 2006. Società Editrice Esculapio, Bologna.

http://www.editrice-esculapio.com/codino-progress-and-prejudice-in-cosmic-ray-p hysics-until-2006/

[11] Boyle, J.P., et al. (2011) New Measurements of the Composition and Energy Spectra of Cosmic-Ray Nuclei with TRACER. Paper 707, 32nd ICRC, Beijing.

[12] Bird, D.J., et al. (1993) Evidence for Correlated Changes in the Spectrum and Composition of Cosmic Rays at extremely High Energy. Phys. Rev. Letters, 71.

[13] Schuessler, F., et al. (2009) The Cosmic Ray Energy Spectrum and Related Measurements with the Pierre Auger Observatory. 31th ICRC, Lodz.

[14] Wiebel-Sooth, B., et al. (2009) Individual Element Spectra: Prediction and Data.

[15] Murphy, R.P., et al. (2015) Abundances of Ultra-Heavy Galactic Cosmic Rays from the Supertiger Instrument. 34th ICRC, Paper 438, Proceedings of Science, Holland.

[16] Binns, W.R., et al. (1989) Abundances of Ultraheavy Elements in the Cosmic Radiation-Results from HEAO-3. The Astrophys Journal, 346, 997. https://doi.org/10.1086/168082

[17] Medina-Tanco, G., et al. (2013) The JEM-EUSO Science Capabilities. 33rd ICRC, Paper 937, Rio de Janeiro.

[18] Salamida, F. (2011) Update of the Measurement of the CR Energy Spectrum above 1018 eV Made Using the Pierre Auger Observatory. 32nd ICRC, Paper 656, Beijing.

[19] Tinyakov, P., et al. (2014) Latest Results from the Telescope Array. Nucl. Instrum. Meth., Section A, 742, 29. 
[20] Jui, C.H. (2000) Results from the High Resolution Fly's Eye Experiment. AIP Conf. Proceeding, 516, 370. https://doi.org/10.1063/1.1291491

[21] Takeda, M., et al. (2003) Energy Determination in the Akeno Giant Air Shower Experiment. Astropar. Physics, 19, 447. https://doi.org/10.1016/S0927-6505(02)00243-8

[22] Bergman, D.R., et al. (2006) Fitting the HiRes Data. Journal of Physics. Conference Series, 47, 154. https://doi.org/10.1088/1742-6596/47/1/019

Submit or recommend next manuscript to SCIRP and we will provide best service for you:

Accepting pre-submission inquiries through Email, Facebook, LinkedIn, Twitter, etc. A wide selection of journals (inclusive of 9 subjects, more than 200 journals)

Providing 24-hour high-quality service

User-friendly online submission system

Fair and swift peer-review system

Efficient typesetting and proofreading procedure

Display of the result of downloads and visits, as well as the number of cited articles Maximum dissemination of your research work

Submit your manuscript at: http://papersubmission.scirp.org/

Or contact jamp@scirp.org 